\title{
Previsão de Tempestades Geomagnéticas Intensas através da observação de raios cósmicos e de componentes do Meio Interplanetário.
}

\author{
Jairo Francisco Savian ${ }^{(1,2)}$, Marlos Rockenbach da Silva ${ }^{(3)}$, Vânia Fátima Andrioli ${ }^{(1,2)}$, Marcos Roberto Signori ${ }^{(1,2)}$, Alisson Dal \\ Lago $^{(3,4)}$, Ezequiel Echer ${ }^{(3)}$, Luis Eduardo Antunes Vieira ${ }^{(3,4)}$, Kazuoki Munakata ${ }^{(5)}$, Walter Demétrio Gonzalez Alarcon ${ }^{(3)}$, \\ Nelson Jorge Schuch ${ }^{(1)}$. \\ (1) Instituto Nacional de Pesquisas Espaciais - Centro Regional Sul de Pesquisas Espaciais - Santa \\ Maria - RS, Brasil - Contato: savian@lacesm.ufsm.br \\ ${ }^{(2)}$ Universidade Federal de Santa Maria - Laboratório de Ciências Espaciais de Santa Maria - Santa \\ Maria, RS, Brasil; \\ ${ }^{(3)}$ Instituto Nacional de Pesquisas Espaciais - Divisão de Geofísica Espacial - São José dos Campos - \\ SP,Brasil; \\ ${ }^{(4)}$ Fundação de Amparo a Pesquisa do Estado de São Paulo - FAPESP - SP, Brasil; \\ ${ }^{(5)}$ Physics Dept., Shinshu University, Nagano, JAPAN.
}

Copyright 2004, SBGf - Sociedade Brasileira de Geofísica

Este texto foi preparado para a apresentação no I Simpósio de Geofísica da Sociedade Brasileira de Geofísica, São Paulo, 26-28 de setembro de 2004. Seu conteúdo foi revisado pela Comissão Tecno-científica do I SR-SBGf mas não necessariamente representa a opinião da SBGf ou de seus associados. E proibida a reprodução total ou parcial deste material para propósitos comerciais sem prévia autorização da SBGf.

\section{Resumo}

Acredita-se que o mecanismo físico responsável pela transferência de energia do Vento Solar para a Magnetosfera seja a reconexão entre o Campo Magnético Interplanetário e o Campo Magnético Terrestre - Tsurutani e Gonzalez, 1997. O critério necessário para que ocorra uma Tempestade Geomagnética Intensa, Dst<-100nT, é o de haver um Campo Elétrico Interplanetário, na direção do anoitecer, maior que $5 \mathrm{mV} / \mathrm{m}$, por um período maior que 3 horas. O objetivo do trabalho é comparar a previsão de Tempestades Geomagnéticas feita por satélites localizados no ponto Lagrangeano - $L_{1}$, e a Rede Internacional de Detectores de Muons, mostrando as vantagens e desvantagens de cada um.

\section{Introdução}

A cada 11 anos o Sol passa por um período de intensa atividade magnética denominado de Máximo Solar, apresentando um maior número de instabilidade as quais liberam grandes quantidades de matérias na forma de Ejeções Coronais de Massa - CME. Estas manifestações de intensa atividade atingem o Meio Interplanetário e os planetas causando as chamadas Tempestades Geomagnéticas. Um parâmetro importante para desenvolvimento das tempestades é a componente "Z" do Campo Magnético Interplanetário - CMI, quando sua direção é antiparalela ao Campo Magnético da Terra na região equatorial ocorre a chamada reconexão magnética, acontecendo um transporte efetivo de energia para a Magnetosfera ocasionando um aumento da densidade de partículas que circundam a Terra. Estes distúrbios solares afeta a população de raios cósmicos galácticos pré-existentes de várias maneiras, a mais conhecida é o "decréscimo de "Forbush", uma região de supressão da densidade de raios cósmicos localizadas atrás de um choque devido a uma Ejeção Coronal de Massa, Figura 1. Dentro da região atrás do choque, anisotropias de gradiente $(B X$ ? $n)$ produzem fluxos de partículas perpendiculares ao campo magnético. Análises destas anisotropias permitem obter o vetor de gradiente de raios cósmicos, o qual contém informações valiosas sobre a estrutura de grande escala e a orientação da ejeção solar. Além disso, na região atrás da onda de choque, fluxos bidirecionais de raios cósmicos podem ser usados como delimitadores da presença de uma ejeção. Os efeitos causados pelas tempestades Geomagnéticas mais conhecidos são: intensificação de correntes elétricas no espaço e na superfície terrestre, ocorrência de auroras nos pólos, aceleração de partículas carregadas, e diversos prejuízos em satélites causando danos no Sistema de Posicionamento Global - GPS, em telecomunicações e até mesmo em astronautas que se encontram em naves espaciais devido a alta radiação emitida. A previsão das Tempestades Geomagnéticas é feita com uma antecedência de duas horas no caso dos satélites e de oito a doze horas utilizando a Rede Internacional de Detectores de Muons.

\section{Metodologia/ Problema Investigado.}

O presente trabalho tem por objetivo apresentar uma análise do evento solar do dia 20 de novembro de 2003 , 
analisando dados do Meio Interplanetário e o estudo das estruturas interplanetárias responsáveis por esta Tempestade Geomagnética Intensa, onde o índice Dst atingiu um valor de $-472 \mathrm{nT}$, sendo uma das maiores Tempestades Geomagnéticas da história. comparamos a previsão feita pelos satélites localizados no ponto Lagrangeano, $L_{1}$, e o decréscimo da contagem de muons feita pela Rede Internacional de Detectors de Muons. Os eventos são analisados através de observações do índice Dst disponíveis no sítio da Universidade de Kyoto Japão, que mede a corrente de anel magnetosférica Mendes Jr., 1992, para identificar as tempestades mais intensas. Em seguida analisamos dados de observações do Meio Interplanetário, preferencialmente do satélite ACE, disponíveis no sistema CDA - Coordinate Data Analise, do Programa ISTP - International SolarTerrestrial Phisics. Para analisar as estruturas responsáveis pelas Tempestades Geomagnéticas foram utilizados raios cósmicos observados através da Rede Internacional de Detectores de Muons, estudadas por Munakata et al. - 2000. O problema investigado é mostrar que é possível prever as Tempestades Geomagnéticas com uma antecedência de uma a duas horas no caso dos satélites e oito a doze horas no caso da Rede Internacional de Detectores de Muons. Desse modo, faz-se necessário um sistema integrado, coletando e analisado dados sobre o espaço, de forma rápida, precisa e confiável para entender os fenômenos físicos relacionados ao Clima Espacial e amenizar os danos tanto econômicos, quanto à vida.

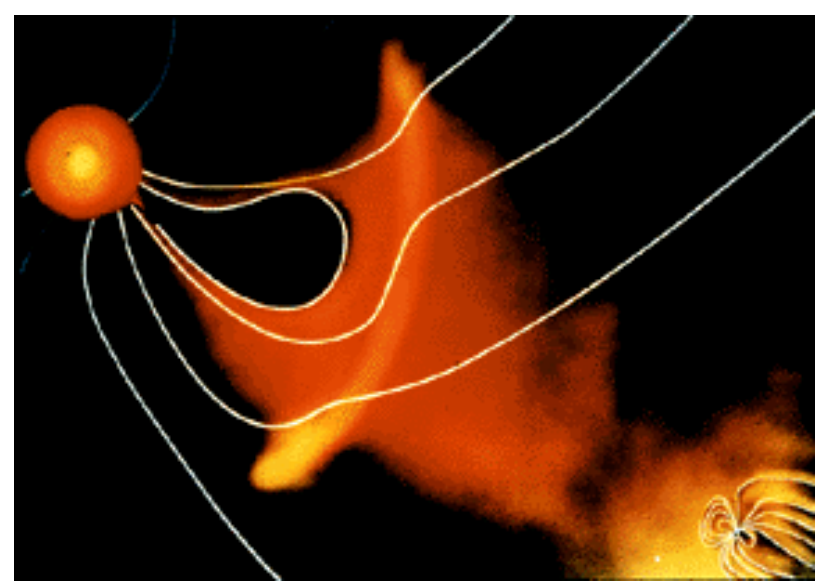

Figura 1 - Interação Sol-Terra mostrando uma Ejeção Coronal de Massa em direção a terra, uma das causas das tempestades Geomagnéticas. Fonte: www.nasa.gov

\section{Resultados}

As 18:48 UT observa-se no disco solar uma explosão "flare" - muito intensa e localizada próximo ao meridiano central do Sol, o que alertou para a possível ocorrência de uma Tempestade Magnética na Terra. A Figura 2 mostra a imagem do instrumento EIT do satélite SOHO, que observa o Sol em comprimentos de onda do ultravioleta extremo - 195 Angstrons, e mostra o momento de máxima emissão desta explosão.

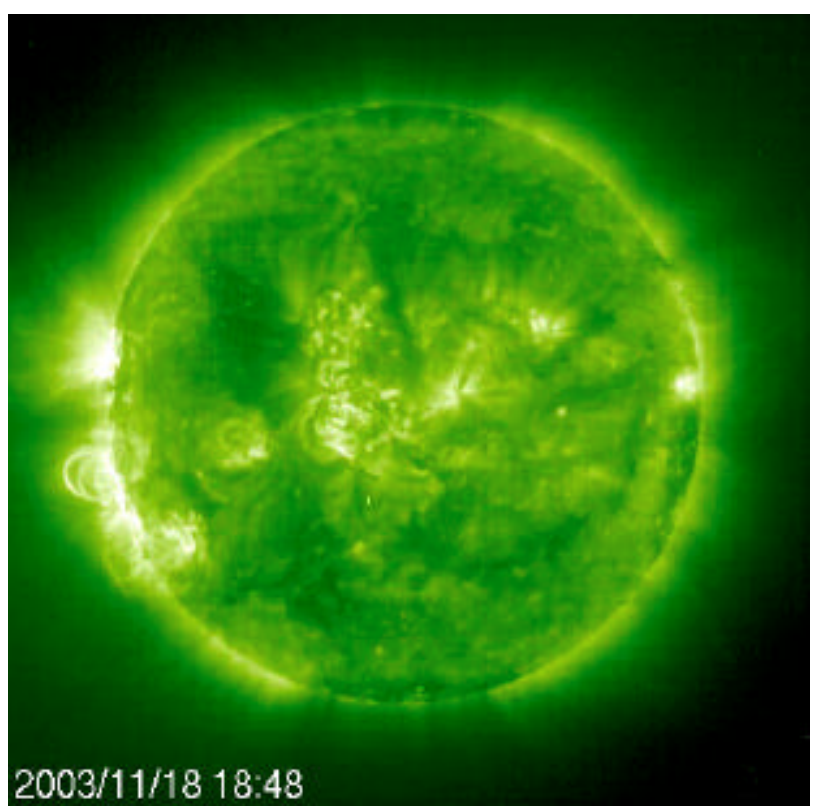

Figura 2 - Flare solar observado em 18/11/2003 pelo satélite SOHO. Fonte: http://star.mpae.gwdg.de

Acompanhando esta explosão, observou-se uma Ejeção Coronal de Massa, através do instrumento LASCO, ver Figura 3, e do satélite SOHO. Esse instrumento oculta o disco solar fazendo um eclipse artificial, permitindo a observação detalhada da coroa solar. Essa ejeção apresentou sob a forma de um "halo" ao redor do Sol, indicando estar sendo ejetada na direção da Terra.

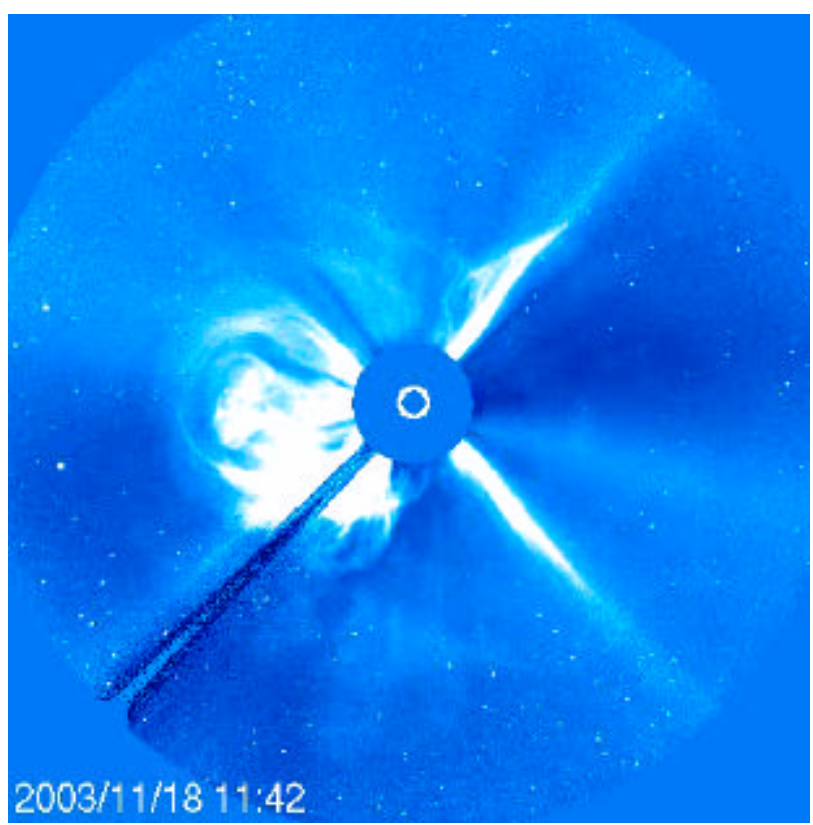

Figura 3 - Ejeção Coronal de Massa tipo halo, observado pelo instrumento LASCO a bordo do satélite SOHO. Fonte: http://star.mpae.gwdg.de 
O decréscimo do índice Dst, sendo identificada como a maior Tempestade Geomagnética do Máximo Solar 23, uma das maiores da história, atingindo um pico de -472 $\mathrm{nT}$, e apresentada na Figura 4.

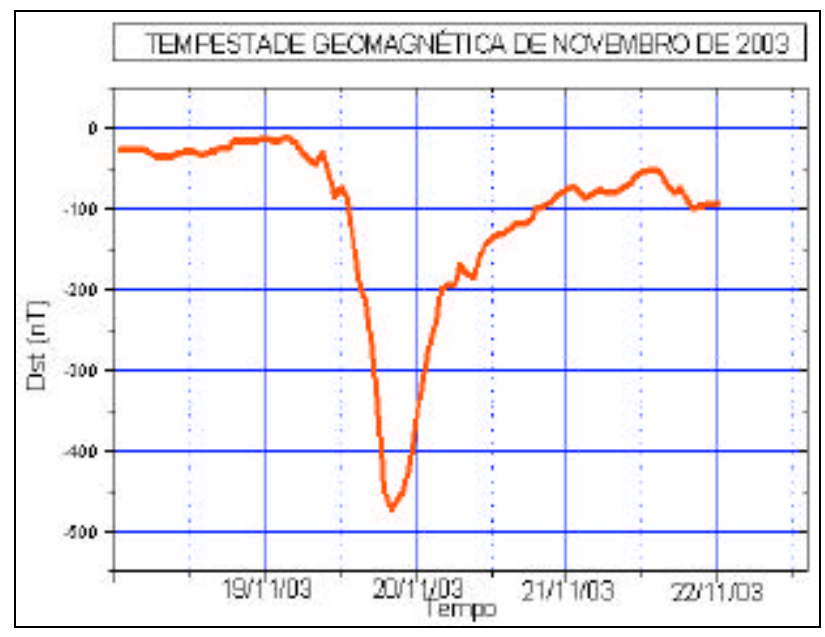

Figura 4 - Índice Dst de 18 à 22 de Novembro de 2003.

No dia 20 de Novembro de 2003 o Telescópio Detector Multidirecional de Muons do Observatório Espacial do Sul OES/CRSPE/INPE-MCT, em São Martinho da Serra, RS, observou um decréscimo na contagem de muons. Este decréscimo foi causado pela Ejeção Coronal de Massa do dia 18 de Novembro de 2003, que devido a onda de choque formada obstrui a passagem de raios cósmicos. A contagem total do Telescópio Multidirecional Detector de muons do Observatório Espacial do Sul , apresentado no topo da Figura 5 , apresenta um decréscimo súbito causado pela onda de choque da Ejeção Coronal de Massa e nos pequenos gráficos abaixo mostra as direções em que se desloca a estrutura. Este decréscimo ocorreu aproximadamente às 21:00 UT.

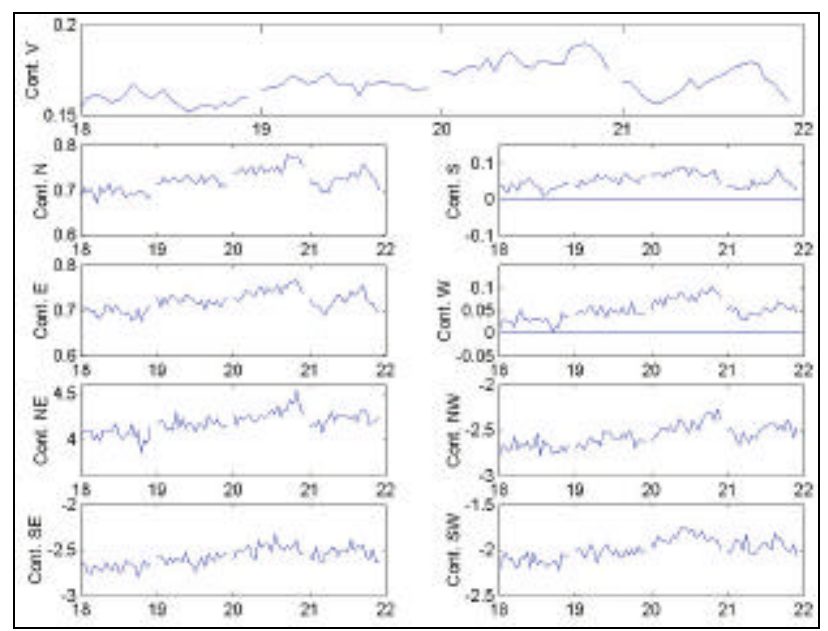

Figura 5 - Decréscimo da contagem de muons do dia 20 de Novembro de 2003.
Dados do satélite ACE - Advanced Composition Explorer - mostram esta chegada, conforme podemos ver na figura 6, que apresenta observações das vizinhanças da Terra, nos dias 19 à 22 de Novembro de 2003. No painel superior está mostrado o campo magnético, o qual tipicamente apresenta valores de $5 \mathrm{nT}$ em períodos calmos, e quando a frente passou após as 6 horas do dia 21 , apresentou valores da ordem de $50 \mathrm{nT}$. Neste mesmo gráfico, mostra, em vermelho, a curva de uma das componentes do campo magnético, a que é a principal responsável pela ocorrência ou não de uma Tempestade Geomagnética na Terra, quando seu valor for negativo, forma-se uma Tempestade Geomagnética.

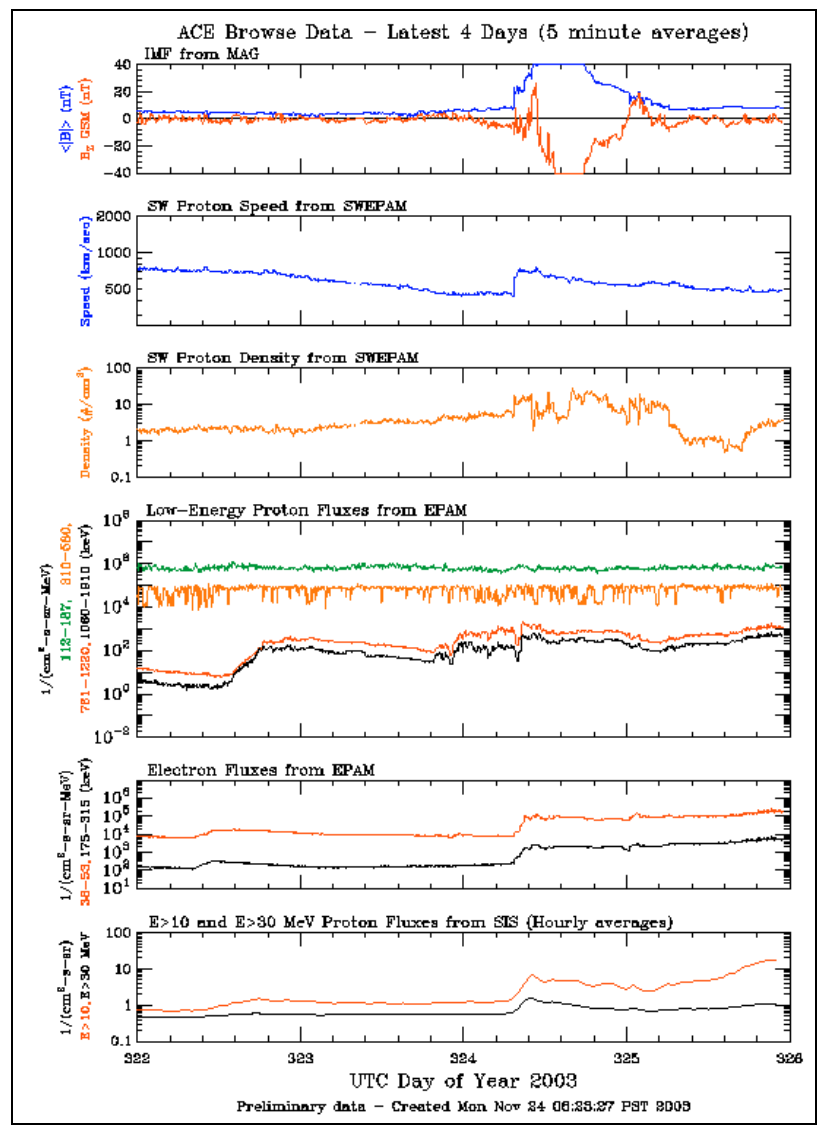

Figura 6 - Dados do satélite ACE. Fonte: http://www.srl.caltech.edu/ACE/ASC/DATA/browseplots/4day_plot_archive/

\section{Discussão e Conclusões}

Após fazermos uma análise completa da Tempestade Geomagnéticas do dia 20 de Novembro de 2003, analisando dados do Meio Interplanetário e de raios cósmicos - muons - vimos a eficiência da utilização da Rede Internacional de Telescópios do Detectores de Muons, devido a sua antecedência na previsão de Tempestades Geomagnéticas em relação a satélites, bem como, a fácil manutenção por estar localizada na superfície de Terra, ao contrário dos satélites. No evento 
do dia 20 de outubro de 2003 prevemos a chegada de uma tempestade com uma antecedência de aproximadamente 9 horas com 0 Telescópio Multidirecional Detector de Muons e de 2 horas no caso dos satélites. $O$ monitoramento contínuo destes parâmetros faz-se necessário para que possamos entender melhor os fenômenos do Meio Interplanetário, o que poderia salvar vários sistemas tecnológicos espaciais ou terrestres.

\section{Agradecimentos}

Os autores gostariam de agradecer ao Dr. Kazuoki Munakata por disponibilizar os dados da Rede Internacional de Telescópios Multidirecionais Detectores de Muons para que fossem analisados e ao pessoal de operação das missões ACE, WINS, IMP-8, bem como ao pessoal de operação de Universidade de Kyoto, por fornecerem os dados interplanetários e de superfície utilizados neste trabalho. Dados do Campo Magnético Interplanetário. Os autores gostariam igualmente de agradecer ao Programa PIBIC/INPE CNPq/MCT e ao programa PET/UFSM - CAPES/MEC pelas bolsas de iniciação científica, e à FAPESP pelos Programas de Pós-Doutoramento, processos 02/14150-0 e 02/12723-2.

\section{Referências}

BURLAGA, L. F.; Interplanetary magnetohydrodynamics. New York: Oxford University Press, 1995, 256p.

BRANT, J. C.; Introduction to the solar wind. San Francisco: W. H. Freeman, 1970. 216p.

COSTA, A.M.da; MENDES JUNIOR, O. Anomalia Magnética do atlântico sul e a radiação cósmica sobre a costa do Brasil. Revista Scientific American Brasil, São Paulo, Ano 2, n. 23, p. 20-29, Abril de 2004.

DAL LAGO. A. Estudo de nuvens magnéticas geoefetivas no meio interplanetário. 1999. 112 p. (INPE-7263-TDI/705. Dissertação (Mestrado em Geofísica Espacial) - Instituto Nacional de Pesquisas Espaciais, São José dos Campos, São Paulo, 1999.

GONZALEZ, W.D; TSURUTANI, B.T. Criteria of interplanetary causing intense magnetic storms(Dst<-100nT). Planet Space Sci, v.35, n.9, p.1101-1109, Jul.1987.

GONZALEZ, W. D; TSURUTANI, B. T; CLUA de GONZALEZ, A. L. Interplanetary origin of geomagnetic storms. Space Sci. Rev. v.88, p.529-562, 1999.

GONZALEZ, W. D.; JOSELYN, J. A.; KAMIDE, Y.; KROEHL, H. W.; ROSTOKER, G.; TSURUTANI, B. T.; E VASYLIUNAS, V. M. What is a magnetic storm?. J. Geophys. Res., v.99, n.A4, p.5771-5792, Apr. 1994.

MUNAKATA, K., BIEBER. J.W., YASUE S.I, KATO C., KOYAMA M., AKAHANE, S., FUJIMOTO, K., FUJII, Z.,
HUMBLE, J.E. e M. L. Duldig, Precursors of geomagnetic storms observed by the muon detector network, J. Geophys. Res., v. 105, n. 27, p. 457, 2000.

RUSSELL, Christopher T.; KIVELSON, Margaret G. Introduction to Space Physics, 1. ed. Los Angeles: Cambridge University Press, 1995.

UNISERSITY OF CALIFORNIA. 